\title{
Region-specific elevations of glutamate + glutamine correlate with the sensory symptoms of autism spectrum disorders
}

\author{
Jason L. He (iD ${ }^{1,2,3,10}$, Georg Oeltzschner ${ }^{1,2,10}$, Mark Mikkelsen ${ }^{1,2}$, Alyssa Deronda ${ }^{4}$, Ashley D. Harris ${ }^{5}$, Deana Crocetti $^{4}$, \\ Ericka L. Wodka ${ }^{6}$, Stewart H. Mostofsky ${ }^{4,7,8}$, Richard A. E. Edden ${ }^{1,2}$ and Nicolaas A. J. Puts (iD $1,2,3,9 \bowtie$
}

(c) The Author(s) 2021

Individuals on the autism spectrum are often reported as being hyper- and/or hyporeactive to sensory input. These sensory symptoms were one of the key observations that led to the development of the altered excitation-inhibition (E-I) model of autism, which posits that an increase ratio of excitatory to inhibitory signaling may explain certain phenotypical expressions of autism spectrum disorders (ASD). While there has been strong support for the altered E-I model of autism, much of the evidence has come from animal models. With regard to in-vivo human studies, evidence for altered E-I balance in ASD come from studies adopting magnetic resonance spectroscopy (MRS). Spectral-edited MRS can be used to provide measures of the levels of GABA + (GABA + macromolecules) and Glx (glutamate + glutamine) in specific brain regions as proxy markers of inhibition and excitation respectively. In the current study, we found region-specific elevations of Glx in the primary sensorimotor cortex (SM1) in ASD. There were no group differences of GABA + in either the SM1 or thalamus. Higher levels of Glx were associated with more parent reported difficulties of sensory hyper- and hyporeactivity, as well as reduced feed-forward inhibition during tactile perception in children with ASD. Critically, the finding of elevated Glx provides strong empirical support for increased excitation in ASD. Our results also provide a clear link between Glx and the sensory symptoms of ASD at both behavioral and perceptual levels.

Translational Psychiatry (2021)11:411 ; https://doi.org/10.1038/s41398-021-01525-1

\section{INTRODUCTION}

While the phenotypical expression of autism spectrum disorders (ASD) is heterogeneous, $295 \%$ of individuals with ASD present as being either hyper- and/or hyporeactive to sensory stimuli [1-4]. Since sensory symptoms can precede the development or presentation of what are considered to be the hallmark characteristics of ASD (i.e., deficits of social communication and restricted, repetitive behaviors) [5], it has been suggested that the pathophysiology underlying these sensory abnormalities may actually drive the development of the other core symptoms of autism [6], though the evidence in support of this suggestion is still being developed and debated. Given the proposed importance of sensory processing to neurodevelopment and functioning in ASD, many studies conducted over the last two decades attempt to both characterize the nature of sensory symptoms of ASD [7-9] and to understand their underlying neurobiological causes $[10,11]$

Investigations of sensory reactivity, or how someone responds to sensory stimuli, have principally relied on the use of self, parent, and/or teacher reports to describe how children with ASD react to a range of stimuli across different sensory domains and contexts (e.g., 'how does your child react to loud noises in their environment?' or 'how does your child feel about wearing certain articles of clothing?') [2, 12-14]. Studies leveraging self, parent, and/or teacher questionnaires to characterize sensory symptoms in ASD have generally found that when compared to children with other neurodevelopmental disorders, as well as typically developing controls (TDC), children with ASD are far more likely to present with symptoms of both hyperreactivity and hyporeactivity, with the evidence for the presence of the latter being more heterogeneous [15]. Interestingly, individuals with ASD who are either hyper- and/or hyporeactive to stimuli in one sensory domain also tend to be similarly hyper- and/or hyporeactive to stimuli in other domains (e.g., a child who is hyperreactive to auditory stimuli also tends to be hyper-reactive to tactile stimuli), suggesting these symptoms to be domain-general rather than domain-specific [16].

Unlike sensory reactivity, which relies on subjective reporting, sensory sensitivity can be assessed objectively using psychophysical methods [17]. Psychophysical paradigms can be used to assess low-level sensitivity (i.e., detection and discrimination) across various perceptual domains [17]. Performance on these paradigms can be typically linked to known neurophysiological processes [18], making inferences from altered sensitivity to

\footnotetext{
${ }^{1}$ Russell H. Morgan Department of Radiology and Radiological Science, The Johns Hopkins University School of Medicine, Baltimore, MD, USA. ${ }^{2} \mathrm{~F}$. M. Kirby Research Center for Functional Brain Imaging, Kennedy Krieger Institute, Baltimore, MD, USA. ${ }^{3}$ Department of Forensic and Neurodevelopmental Sciences, Sackler Institute for Translational Neurodevelopment, Institute of Psychiatry, Psychology, and Neuroscience, King's College London, London, UK. ${ }^{4}$ Center for Neurodevelopmental and Imaging Research, Kennedy Krieger Institute, Baltimore, MD, USA. ${ }^{5}$ Department of Radiology, University of Calgary, Calgary, Canada. ${ }^{6}$ Center for Autism and Related Disorders, Kennedy Krieger Institute, Baltimore, MD, USA. ${ }^{7}$ Department of Neurology, The Johns Hopkins University School of Medicine, Baltimore, MD, USA. ${ }^{8}$ Department of Psychiatry and Behavioral Sciences, The Johns Hopkins University School of Medicine, Baltimore, MD, USA. ${ }^{9}$ MRC Centre for Neurodevelopmental Disorders, King's College London, London, UK. ${ }^{10}$ These authors contributed equally: Jason L. He, Georg Oeltzschner. ${ }^{凶}$ email: Nicolaas.puts@kcl.ac.uk
}

Received: 8 March 2021 Revised: 4 June 2021 Accepted: 10 June 2021

Published online: 29 July 2021 
abnormal neurophysiological functioning possible. Through psychophysics, we and others have observed patterns of altered sensory sensitivity in ASD that were broadly consistent with reduced or inefficient inhibitory signaling [19-28]. For instance, our own work conducted in the tactile domain revealed that children with ASD showed less of an increase in detection thresholds when perceiving stimuli of increasing intensity and frequency. Indeed, this pattern is consistent with reduced thalamocortical feed-forward inhibition and altered GABAergic signaling [29-32]. Further, we recently found that children with ASD, as opposed to children with attention-deficit hyperactivity disorder (ADHD), have particular difficulties with discriminating between the amplitude and frequency of sequentially or simultaneously delivered tactile stimuli [16]. Likewise, these difficulties point to altered lateral inhibition, a process that helps sharpen receptive fields for enhanced discrimination, and as with feed-forward inhibition, is also principally dependent on GABA. Importantly, our findings in the tactile domain parallel similar findings in both auditory [33] and visual [34] domains, again highlighting the domain-generality of sensory symptoms in ASD.

When taken together, the evidence from investigations of both sensory reactivity and perceptual sensitivity in ASD are beginning to suggest that being hyper- and hyporeactive to sensory input originates from alterations at the perceptual level. More critically, since both feed-forward inhibition and lateral inhibition are dependent on GABAergic processes [35-38], results from these psychophysical experiments further suggest that altered sensory perception in ASD is, at least in part, due to reduced or inefficient GABAergic functioning in ASD. Indeed, the idea of abnormal GABA in ASD is not novel and there are many other lines of supporting evidence [39]. Since its original proposal by Hussman [40], and the proliferation of the idea through the frequently cited review article by Rubenstein and Merzenich [41], studies conducted from various levels of analysis have found support for the altered excitation-inhibition (E-I) balance theory of ASD, which suggests that the symptoms of autism can be explained by a shift in the overall balance of glutamatergic 'excitatory' and GABAergic 'inhibitory' signaling [42].

The role of E-I balance in ASD has been investigated in both humans and in animal models [43]. While animal-based work can provide important insights into the pathophysiology of E-I balance in autism, due to the complexity of behavioral symptom presentation of ASD, animal-based work cannot capture the associations between neurobiological alterations and behavior as well as human studies. Magnetic resonance spectroscopy (MRS) is a non-invasive technique that allows for the measurement of neurometabolite levels in-vivo in both humans and animals [44]. Edited MRS can be used to measure the levels of neurometabolites that would otherwise be difficult to detect at low field strengths [45]. Of relevance to the current study, edited MRS can be used to measure the levels of GABA and glutamate (note that GABA measures from spectral editing are contaminated by overlapping macromolecular signals and glutamate is difficult to resolve from the similar glutamine, hence GABA and glutamate levels acquired by MRS are typically referred to as GABA+ and Glx [glutamate + glutamine], respectively [46]).

Findings from studies applying edited-MRS to assess GABA+ and glutamate in ASD have been rather inconsistent [47], perhaps owing to the heterogeneity of the condition itself, but also to the variety of MRS methods, brain regions, and age cohorts represented across these studies [48]. In the current study, we focused on Glx and GABA + levels, interpreting them as broad surrogate markers of excitation and inhibition in regions specifically involved in tactile processing: the primary sensorimotor cortex (SM1) [49-51] and the thalamus (Thal) [52-54]. First, we compared relative concentrations of GABA+ and Glx in SM1 and Thal between ASD and controls, hypothesizing that GABA + would be decreased in ASD (as in our prior work [30]), and making no specific directional hypothesis regarding Glx. We then conducted exploratory analyses to investigate the associations between Glx and GABA + to parent reports of sensory reactivity and psychophysically derived measures of tactile sensitivity. With these exploratory analyses, we broadly hypothesized that lower GABA+ and higher Glx levels would be associated with both greater sensory hyper- and hyporeactivity and lower tactile sensitivity (e.g., higher perceptual thresholds and reduced feedforward inhibition).

\section{MATERIALS AND METHODS}

\section{Enrollment and clinical assessment}

In all, 73 children with ASD and 92 TDCs were enrolled to participate in this study (see Table 1 for demographics). A parent of each child assented to testing and provided written informed consent to their child participating. The study protocol was approved by the local institutional review boards of the Kennedy Krieger Institute and The Johns Hopkins University School of Medicine.

Participants with ASD met diagnostic criteria based on the Diagnostic and Statistical Manual of Mental Disorders Fifth Edition (DSM-5) criteria [55] and was verified using the Autism Diagnostic Observation Schedule Second Edition (ADOS-2) [56] and Autism Diagnostic Interview-Revised (ADI-R) [57]. The Wechsler Intelligence Scale for Children - Fourth (WISC-IV) [58] and Fifth (WISC-V) [59] editions were used to determine general cognitive and intellectual ability. Handedness was determined using the Edinburgh Handedness Inventory [60].

Children with identifiable genetic causes of autism (e.g., Fragile $X$ syndrome) and other neurological disorders were excluded. Children with full-scale IQ scores below 80 were excluded from participation unless there was a 12-point or greater index discrepancy, in which case either the Verbal Comprehension Index or perceptual reasoning index (PRI) was required to be $\geq 80$ and the lower of the two was required to be $\geq 65$.

Participants in the ASD cohort were instructed to discontinue stimulant medication on the day of participation, as well as the day before, but were allowed to take other psychotropic medications requiring extended washout. None of the children in the TDC cohort were prescribed psychoactive medications.

\section{MRS of GABA + and Glx}

Acquisition. All structural MRI and MRS data were acquired on a Philips 3T MRI scanner (Philips Healthcare, Best, The Netherlands). During the data collection period, the MRI scanner underwent hardware and software upgrades. Our methodological research in the field of edited MRS also saw improvements in the acquisition parameters of the protocol initially selected for acquiring GABA+ and Glx. Thus, data were collected in three separate acquisition phases (the parameters of which are described in detail in the Supplementary Methods).

Following a fast survey image, a high-resolution $\left(1 \mathrm{~mm}^{3}\right.$ isotropic) $\mathrm{T}_{1}$ weighted (MP-RAGE) image was acquired to guide voxel placement and to be used for tissue segmentation in subsequent data processing. In all phases (labeled 1 to 3$)$, an isotropic MRS voxel $\left(30 \times 30 \times 30 \mathrm{~mm}^{3}[27 \mathrm{ml}]\right)$ was placed in the right primary sensorimotor cortex (SM1). The voxel was centered using the hand-knob in the central sulcus as a guiding anatomical landmark [61] and was rotated to be aligned with the dorsolateral surface (see Fig. 1a). For phases 2 and 3, an additional MRS voxel $(26 \mathrm{~mm}(A P) \times 24 \mathrm{~mm}(C C) \times 40 \mathrm{~mm}(\mathrm{LR})[25 \mathrm{ml}])$ was placed in the thalamus (Thal). This voxel was positioned so as to include both halves of the thalamus, sacrificing information on laterality to achieve sufficient signal-to-noise ratio (Fig. 1b). Common parameters across all phases were: 320 averages per voxel; TR/TE $=2000 / 80$ ms; 2048 samples; 2 kHz spectral width; and VAPOR water suppression [62].

Preprocessing and quantification. A comprehensive description of how data were preprocessed and quantified is provided in Supplementary Methods. Briefly, metabolite concentrations were estimated using Gannet (version 3.1), a software package developed for the automated batch processing of edited MRS data [63]. Using tissue segmentation implemented in SPM12 [64], the fractional tissue volumes for gray matter (GM), white matter (WM), and cerebrospinal fluid (CSF) were determined for each voxel of the $T_{1}$-weighted image acquired at the start of the scan session. $\mathrm{GABA}+$ and Glx measurements were then corrected for interindividual differences in voxel compositions of GM, WM, and CSF to increase 
Table 1. Descriptive statistics for demographic variables and key clinical outcomes.

\begin{tabular}{|c|c|c|c|c|c|c|c|}
\hline & \multicolumn{3}{|l|}{ ASD } & \multicolumn{4}{|l|}{ TDC } \\
\hline & $N$ & $M$ & SD & $N$ & $M$ & SD & $p$ \\
\hline \multicolumn{8}{|l|}{ Demographics } \\
\hline $\operatorname{Sex} M(F)$ & $42(2)$ & - & - & $46(16)$ & - & - & 0.009 \\
\hline Handedness R (L) & $40(4)$ & - & - & $57(5)$ & - & - & $>0.999$ \\
\hline WISC4 - FSIQ & 3 & 102.33 & 4.04 & 13 & 114.69 & 7.59 & 0.017 \\
\hline \multicolumn{8}{|l|}{ ADOS } \\
\hline Social interaction & 44 & 7.36 & 3.58 & 61 & - & - & - \\
\hline Communication & 44 & 3.25 & 1.91 & 61 & - & - & - \\
\hline Stereotyped & 44 & 2.7 & 1.82 & 61 & - & - & - \\
\hline Total & 44 & 13.32 & 6.6 & 61 & - & - & - \\
\hline SEQ sensory seeking & 37 & 2.47 & 0.6 & 40 & 1.45 & 0.4 & $<0.001$ \\
\hline \multicolumn{8}{|c|}{ MRS metabolite levels (residuals after modeling effects of acquisition phase) } \\
\hline \multicolumn{8}{|c|}{ SM1 } \\
\hline $\mathrm{Glx} / \mathrm{Cr}$ & 44 & 0.00556 & 0.01319 & 59 & -0.00415 & 0.01815 & - \\
\hline Glx (IU) & 44 & 0.68312 & 1.59195 & 59 & -0.50944 & 1.68914 & - \\
\hline $\mathrm{GABA}+/ \mathrm{Cr}$ & 42 & 0.00022 & 0.01226 & 53 & -0.00018 & 0.01056 & - \\
\hline $\mathrm{GABA}+(\mathrm{IU})$ & 41 & 0.03637 & 0.33894 & 54 & -0.02761 & 0.31498 & - \\
\hline \multicolumn{8}{|l|}{ Thal } \\
\hline $\mathrm{Glx} / \mathrm{Cr}$ & 20 & 0.00049 & 0.00524 & 26 & -0.00037 & 0.00662 & - \\
\hline Glx (IU) & 18 & 0.0095 & 0.69202 & 29 & -0.0059 & 0.76507 & - \\
\hline
\end{tabular}

MRS descriptive statistics are referring to the mean (M) and standard deviation (SD) of the residuals from the models used to combine across acquisition phases. Note: categorical variables (sex and handedness) were compared using $x^{2}$ test of independence. All other variables were compared using independent samples t-tests. "-" - MRS descriptive statistics are not compared here, see main text.

$\mathrm{N}=$ Number of participants, $M=$ Mean, $\mathrm{SD}=$ Standard Deviation, WISC - Wechsler Intelligence Scale - Children, $\mathrm{FSIQ}=\mathrm{Full} \mathrm{Scale} I \mathrm{IQ}, \mathrm{ADOS}=$ Autism Diagnostic Observation Scale, ADI = Autism Diagnostic Interview; SEQ = Sensory Experience Questionnaire, SM1 = sensorimotor cortex, Thal = thalamus, $\mathrm{Glx}=$ glutamate + glutamine, $\mathrm{Cr}=$ creatine, $\mathrm{IU}=$ institutional units.

a

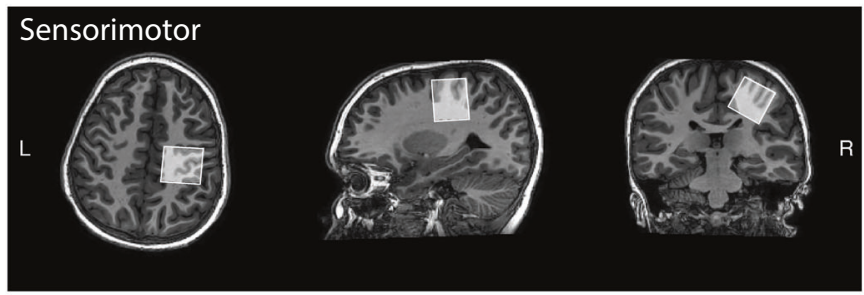

C

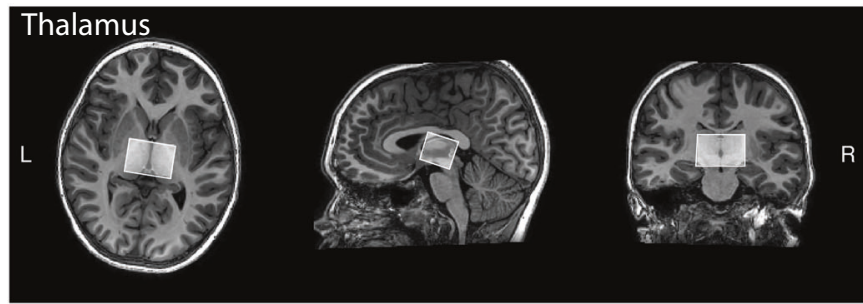

b

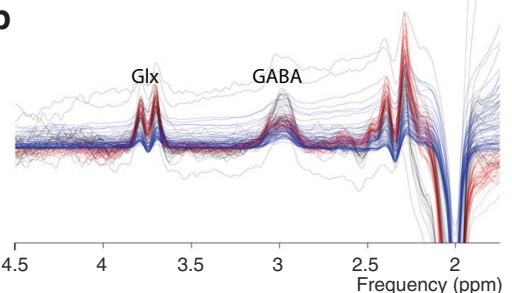

d

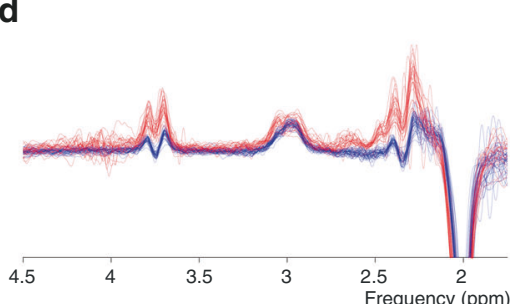

Fig. 1 Voxel placement and example spectra. a Depiction of voxel placement for the left primary sensorimotor cortex (SM1) in a randomly selected participant. b Spectra from all the participants, color coded by the acquisition phase from which they were collected (black = phase 1 , blue $=$ phase 2, and red = phase 3 - see Supplementary Methods). c Voxel placement for the thalamus (Thal) in a randomly selected participant. d Spectra from all participants, color coded by acquisition phase. Note: no phase 1 data were acquired in the Thal. 
precision and accuracy of quantification $[65,66]$. Metabolite estimates are typically referenced to either tissue water or total creatine [45]. To account for the possibility that our results were driven by interindividual differences in the reference (i.e., either tissue water or total creatine) rather than our metabolites of interest, GABA+ and Glx estimates were quantified relative to both water (alpha-tissue corrected, referred to as GABA + (IU) and Glx (IU)) and creatine (referred to as GABA $+/ \mathrm{Cr}$ and $\mathrm{Glx} / \mathrm{Cr}$ ).

Data quality was visually inspected by GO ( $\sim 9$ years of experience using edited MRS). Individual fits were excluded from analyses if either the data themselves or the fits were considered unusable (e.g., due to excessive subject motion), resulting in strongly diminished spectral quality (e.g., subtraction artefacts, or a degree of lipid contamination in cortical voxels strong enough to interfere with the peak modeling). Finally, estimates across the acquisition phases were compared (see Supplementary Table 1) and combined using linear mixed-effect models treating acquisition phase as a random factor. The residual values for each participant (i.e., the variance not accounted for by acquisition phase) were then used as estimates of GABA+ and Glx. For reasons of readability, we continue to refer to these variables as GABA + (IU), Glx (IU), GABA+/Cr, and $\mathrm{Glx} / \mathrm{Cr}$ rather than referring to them as residuals (e.g., residual GABA + (IU)).

\section{Sensory reactivity and sensitivity}

Sensory Experience Questionnaire. Sensory reactivity was assessed using the Sensory Experience Questionnaire (SEQ) [2]. The SEQ is intended to be completed by parents to assess how their child reacts to everyday sensory experiences across different sensory domains. The SEQ was specifically designed to characterize sensory features in young children with ASD and discriminates between patterns of hyper- and hyporeactivity, as well as sensory seeking behaviors (described as craving of certain sensory stimuli, perhaps as a way to self-regulate stimulation levels [67]) among children with ASD, children with other developmental disorders and TDCs. Questions such as 'how often does your child react sensitively to unexpected or loud sounds?' or 'how often does your child seem slow to look at objects that are placed or held near him/her' are used to probe sensory hyper- and hyporeactivity respectively. Similarly, questions such as how often does your child: 'smell objects or toys during play or other activities' are used to probe sensory seeking behaviors. Total item scores from the SEQ were used for subsequent analysis.

Tactile sensitivity. Perceptual sensitivity was assessed in the tactile domain using a battery of vibrotactile tasks (total duration $\sim 40$ mins) [68]. Participants were instructed to rest their left hand on a CM4 four-digit tactile stimulator (Cortical Metrics, Carrboro, NC). The stimulator was used to deliver vibrotactile stimuli within the flutter range $(25-50 \mathrm{~Hz})$ to the glabrous skin on digit 2 (LD2 - index finger) and digit 3 (LD3 - middle finger) of the left hand via two cylindrical plastic probes with $5 \mathrm{~mm}$ diameter. The battery consisted of 11 tasks, grouped into five domains (reaction time, detection threshold, amplitude discrimination, frequency discrimination, and temporal order judgment), with three conditions in the amplitude discrimination domain and two conditions for each of the other domains. For brevity, we focus on the tasks that have previously revealed group differences between ASD and TDCs $[16,19]$, namely the static and dynamic detection, and amplitude and frequency discrimination paradigms. See Fig. 2 and Supplementary Methods for further details.

\section{Statistical analysis}

All statistical analyses were conducted using the $\mathrm{R}$ programming language (version 4.0.2) in R Studio (version 1.2.1335) [69]. The code used to generate the results and figures of this manuscript are available online through the Open Science Framework (https://osf.io/xhqwu/). Alpha levels for all analyses were set to 0.05 . Where relevant, partial eta-squared $\left(\eta_{p}{ }^{2}\right)$ was estimated using the 'effectsize' package [70]. Bayes factors were estimated using the 'BayesFactor' package [71] using non-informative Jeffreys's priors [72]. Statistical outliers for all relevant variables were a

b
C

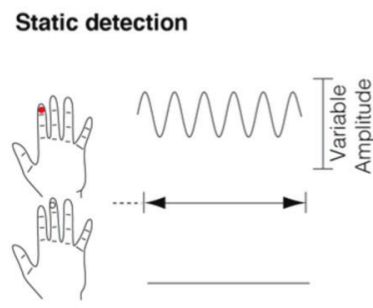

d

Simultaneous frequency discrimination

Standard

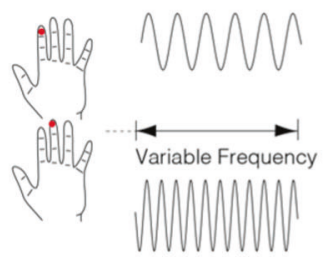

Comparison
Dynamic detection

Respond when perceived

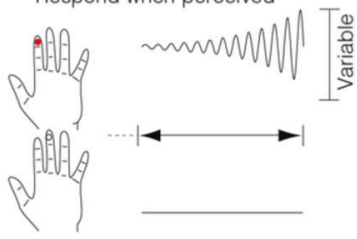

e

Sequential frequency discrimination

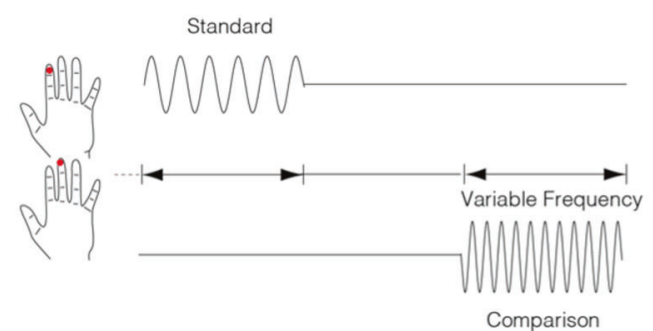

Fig. 2 Visual schematic of relevant tasks from the vibrotactile battery. a Static detection protocol in which participants must detect which finger (left digit 1 or left digit 2) received the stimulation. Stimuli are presented with a fixed frequency and an amplitude that is increased or decreased based on incorrect and correct responses respectively. b Dynamic detection protocol in which participants are instructed to respond immediately after they perceive a stimulus of increasing amplitude. c Amplitude discrimination protocol in which a standard stimulus with a fixed amplitude and frequency is compared to a comparison stimulus in which the amplitude varies based on performance (i.e., the difference in amplitudes between the standard and comparison stimulus is increased following an incorrect response but decreases following an incorrect response). d Simultaneous frequency discrimination, similar to the amplitude discrimination protocol but rather than the stimulus amplitude changing based on performance, frequency of the comparison stimulus changes instead. e Sequential frequency discrimination, like the simultaneous frequency discrimination but stimuli are delivered sequentially (requiring less lateral inhibition and likely requires more accurate temporary storage of stimulus characteristic for later comparison). Full details about the parameters and timing of the stimuli are provided in Supplementary Methods. 
removed using the median absolute deviation method using a 2.5 standard deviation threshold [73].

Quantitative MRS data quality was first assessed using independent samples $t$-tests comparing fit values and full-width half maximum (FWHM) of the modeled NAA signal in the non-edited spectrum for each acquisition phase (see Supplementary Table 2). To determine whether there were group differences in GABA + and Glx levels for both regions of interest, a $2 \times 2$ repeated-measures ANOVA was conducted to compare GABA + and Glx levels between Region (SM1 and Thal) and Group (ASD and TDC). To determine whether interindividual differences in GABA+ and GIx in both SM1 and Thal were significantly related to sensory reactivity, we conducted Pearson's correlation analyses between GABA + and Glx from SM1 and Thal to total sensory hyper- and hyporeactivity, and sensory seeking scores from the SEQ. Similarly, to determine whether neurometabolite levels in our regions of interest were significantly related to sensory sensitivity, we conducted Pearson's correlation analyses between GABA + and GIx of SM1 and Thal to perceptual thresholds measured through the vibrotactile psychophysical battery. All correlations were first conducted across the entire sample (i.e., considering ASD and TDC participants together) before considering whether there was a moderating effect of Group.

Additional exploratory analyses required for interpretation of our results are described in-line below. The sample size presented here is larger than many existing studies (including our own work) which have identified group differences or correlations using MRS-derived measures of excitation and inhibition. Bonferroni's method was applied to analyses that were (a) conducted following a significant interaction effect (each $p$-value was multiplied by the number of slopes analyzed), or (b) involved multiple correlation analyses between a given metabolite and multiple scale items on a questionnaire (each $p$-value was multiplied by the number of associations conducted between each metabolite and each item of each questionnaire). Alpha was set 0.05 and all tests conducted were two-sided.

\section{RESULTS}

Descriptive statistics for all relevant variables in the subsequent analyses below are presented in Table 1. Descriptive statistics for performance on the tactile protocols are presented in Supplementary Table 3. Results for the analyses conducted on Glx and GABA + referenced to creatine are presented in Supplementary Results. All instances of $M$ and SD presented in-text refer to mean and standard deviations.

\section{Comparing metabolite levels of SM1 and Thal between ASD and TDCs}

Elevated levels of Glx in SM1 but not Thal in ASD. While there was very strong evidence for a significant main effect of Group on Glx (IU) levels $\left[F(1,146)=12.27, p<0.001 ; \eta_{\mathrm{p}}{ }^{2}=0.078 ; \mathrm{BF}_{10}=38.16\right]$, there was also substantial evidence for a Group by Region interaction effect $\left[F(1,146)=5.22, p=0.024 ; \eta_{\mathrm{p}}{ }^{2}=0.034 ; \mathrm{BF}_{10}=\right.$ 7.67] (Fig. 3a). Subsequent simple main effect analyses found strong evidence for an effect of Group on SM1 Glx (IU) $[F(1,101)=$ 13.19, $\left.p_{\text {Bonferroni }}<0.001 ; \eta_{\mathrm{p}}{ }^{2}=0.116 ; \mathrm{BF}_{10}=59.48\right]$, which was absent for Thal $\left[F(1,45)=0.00, p_{\text {Bonferroni }}=0.999 ; \eta_{\mathrm{p}}{ }^{2}=0.000\right.$; $\left.\mathrm{BF}_{10}=0.30\right]$. Indeed, although children in the ASD $(M=0.68, \mathrm{SD}=$ 1.59) group had higher SM1 Glx levels compared to the TDC group $(M=-0.01, \mathrm{SD}=0.69)$, the groups had otherwise comparable Thal Glx levels [ASD: $M=0.01, S D=0.69$; TDC: $-0.01, S D=0.77$ ] (see Fig. 3b, c). A similar pattern of results was observed when Glx was referenced to creatine (see Supplementary Results and Supplementary Fig. 1), suggesting that the pattern of these effects was not driven by the reference signal.

Comparable levels of GABA+ in SM1 and Thal between ASD and TDCs. In contrast to Glx, there was evidence against a main effect of both Group $\left[F(1,148)=2.14, p=0.146, \eta_{\mathrm{p}}{ }^{2}=0.014 ; \mathrm{BF}_{10}=0.47\right]$ and Region $\left[F(1,148)=0.00, p=0.972 ; \eta_{\mathrm{p}}{ }^{2}=0.022 ; \mathrm{BF}_{10}=0.18\right]$ on GABA + levels. There was also evidence against an interaction effect $\left[F(1,148)=0.52, \quad p=0.474 ; \quad \eta_{\mathrm{p}}{ }^{2}=0.003 ; \quad \mathrm{BF}_{10}=0.08\right]$ (Fig. 3d). Indeed, SM1 GABA+ levels were comparable between ASD and TDC groups for SM1 [(ASD: $M=0.04$, SD $=0.34$; TDC: $M=-0.03, \mathrm{SD}=0.31)]$ and Thal $[(\mathrm{ASD}: M=0.09, \mathrm{SD}=0.63$; TDC:
$M=-0.08, \mathrm{SD}=0.51$ )] (Fig. 3e, f). As with Glx, the same pattern of results was observed for $\mathrm{GABA}+/ \mathrm{Cr}$.

Between-region correlation of metabolites: evidence for increased glutamatergic thalamocortical connectivity in ASD. Altered thalamocortical connectivity in ASD has long been suggested [74-76]. While MRS can only provide region-specific estimates of neurometabolite levels, it is possible to assess how neurometabolites levels are associated across regions, as well as whether and how those associations differ between groups. Given that thalamocortical relay neurons are predominantly glutamatergic [77], correlations between Glx levels of SM1 and Thal could be associated with thalamocortical connectivity.

As an indirect assessment of thalamocortical connectivity, we compared the correlation between SM1 Glx and Thal Glx between ASD and controls. While there was a significant positive correlation between SM1 Glx and Thal Glx across the whole sample [ $r=0.42$, $\left.\left.F(1,54)=11.45, p=0.001 ; \eta_{\mathrm{p}}{ }^{2}=0.18 ; \mathrm{BF}_{10}=28.70\right)\right]$, there was very strong evidence towards a moderating effect of Group $[(F(1$, $\left.52)=5.72, p=0.006) ; \eta_{p}{ }^{2}=0.08 ; B_{10}=44.34\right]$. Subsequent simple slope analyses revealed that while there was no meaningful association between SM1 Glx and Thal Glx in the TDC group ( $r=$ $0.01, p_{\text {Bonferroni }}=0.999$ ), there was a moderate to strong positive correlation in the ASD group ( $r=0.55, p_{\text {Bonferroni }}=0.007$ ) (Fig. $3 g$ ). These results provide indirect support for increased thalamocortical connectivity in ASD compared to controls. For completion, we also assessed inter-region associations of GABA+. There was no correlation between SM1 GABA + and Thal GABA $+[(r=-0.15, F(1$, $\left.\left.52)=1.15, p=0.288 ; \eta_{\mathrm{p}}{ }^{2}=0.02 ; \mathrm{BF}_{10}=0.44\right)\right]$. There was also no moderating effect of Group on the associations between SM1 and Thal GABA $\left.+\left[F(1,51)=0.68, p=0.415 ; \eta_{\mathrm{p}}{ }^{2}=0.01 ; \mathrm{BF}_{10}=0.16\right)\right]$. See Fig. 3h. The same pattern of effects were observed for $\mathrm{Glx} / \mathrm{Cr}$ and GABA+/Cr (see Supplementary Fig. 2).

\section{Correlations between metabolite levels of SM1 and Thal with sensory reactivity}

SM1 Glx is associated with both hyper- and hyporeactivity. There were significant associations between SM1 Glx and parent reported hyper- $\left(r=0.36, p_{\text {Bonferroni }}=0.005\right.$; Fig. $\left.4 a\right)$ and hyporeactivity $\left(r_{\text {Bonferroni }}=0.32, p_{\text {Bonferroni }}=0.015\right.$; Fig. $\left.4 b\right)$ on the SEQ. There was no meaningful association between SM1 Glx and sensory seeking $\left(r=0.11, p_{\text {Bonferroni }}=0.999\right)$. There were no moderating effects of Group for any of these associations (all $p_{\text {Bonferroni }}>0.999$ ). We recognize that the associations between SM1 Glx with hyper- and hyporeactivity can be interpreted as being driven by clustering within the groups. Typically, when data is clustered within groups of a correlation, it is argued that the correlation is being driven by group differences, rather than reflecting a true correlation. Indeed, given the group differences on both SEQ hyper- $(p<0.001)$ and hyporeactivity $(p<0.001)$, as well as SM1 Glx (see Fig. 3b), the data points are clustered in such a way that the children in the ASD group are clustered towards the top right and children in the TDC group are clustered towards the bottom. While it is the case that group differences leading to clustered data can drive spurious correlations [78], we argue that this is only an issue when it results in a bimodal distribution for one or both of the variables being correlated. Given that SEQ hyper- and hyporeactivity, SEQ sensory seeking, SM1 Glx and Thal GABA (i.e., the variables in the associations presented in Fig. $4 a-c$ ) are not bimodally distributed and are approximately normal (see Supplementary Figs. 3 and 4), we believe these associations reflect true associations between hyper- and hyporeactivity with SM1 GIx levels. That said, existence of a clear sub-cluster of those with ASD with high Glx that show high SEQ scores could be indicative of a specific phenotype with potential clear biological markers. There were no associations between Thal Glx with any of the total scores from the SEQ (all $p_{\text {Bonferroni }}>0.999$ ). There were also no moderating effects of Group (all $p_{\text {Bonferroni }}>0.999$ ). 
a

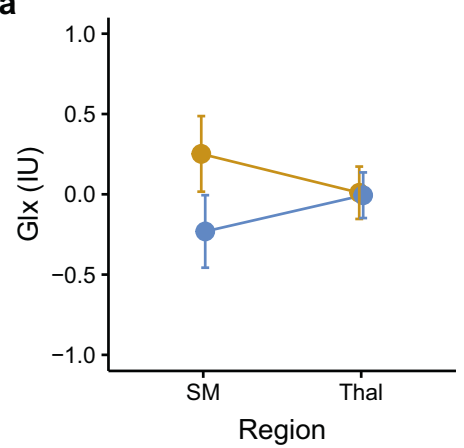

d

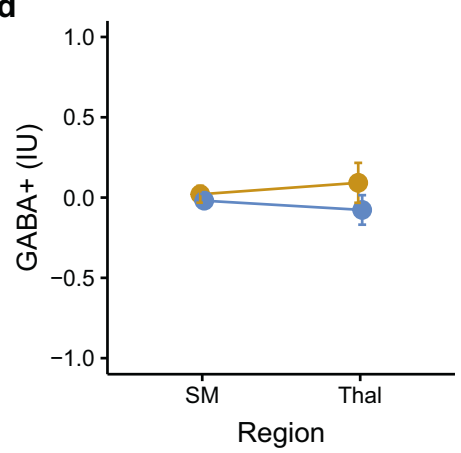

g

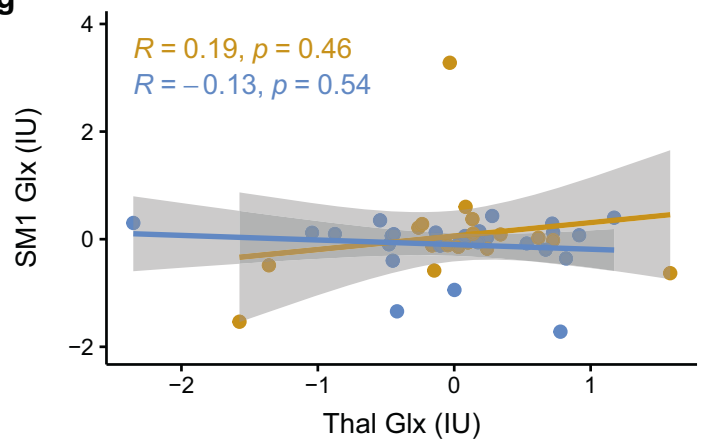

b
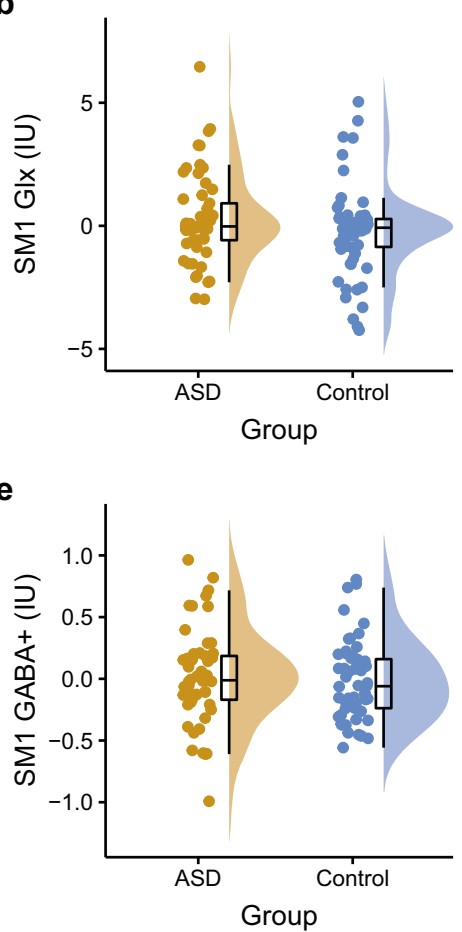

C

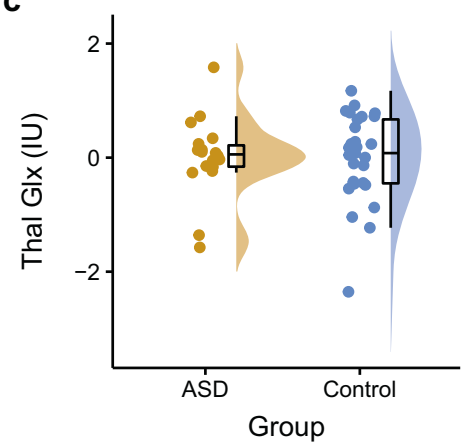

f

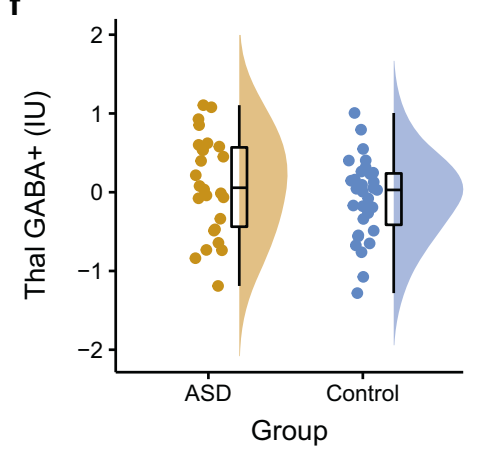

h

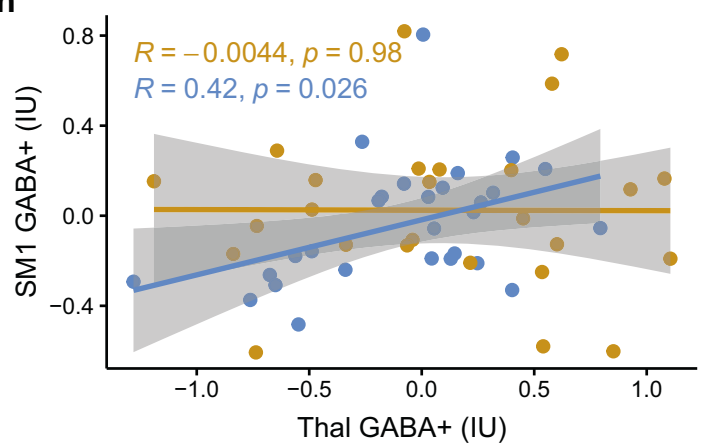

Fig. 3 Region-specific increase in Glx but not GABA+ in ASD. The ASD and TDC group are depicted as yellow and blue (respectively). a Evidence towards a Group by Region interaction effect on Glx (IU). b Higher Glx (IU) levels in the SM1 voxel for children in the ASD compared to TDC group. c Low evidence towards a meaningful group difference in Glx (IU) levels in the Thal voxel. d Lack of evidence towards a significant Group by Region interaction effect on GABA + (IU) levels. e Comparable GABA + (IU) in SM and f Thal voxels between children in the ASD and TDC groups. $\mathbf{g}$ Linear relationship between SM Glx and Thal Glx in ASD that is otherwise absent in TDC. $\mathbf{h}$ No relationship between SM GABA + and Thal GABA + in either ASD or TDC. IU institutional units, Glx glutamate + glutamine, Cr creatine, SM1 primary sensorimotor cortex, Thal thalamus, ASD autism spectrum disorders, TDC typically developing controls. Note: $p$-values presented on panels $\mathbf{g}$ and $\mathbf{h}$ are not corrected for multiple comparisons, please refer to the $p$-values in the main text. Error bars in panels a and $\mathbf{d}$ represent standard error. The shaded area around the line of best fit represents the $95 \%$ confidence interval.

Thal GABA+ is associated with sensory seeking behaviors. There were no significant associations between SM1 GABA+ and any of the total scores on the SEQ (all $p_{\text {Bonferroni }}>0.060$ ). There was also no significant moderating effect of Group (all $p_{\text {Bonferroni }}>0.969$ ).

There was a trend towards an association between Thal GABA+ and parent reported sensory seeking total scores $(r=0.34$, $p_{\text {Bonferroni }}=0.054$; Fig. $4 \mathrm{c}$ ). There was otherwise no meaningful association between Thal GABA+ with either hyper- $(r=0.21$, $\left.p_{\text {Bonferroni }}=0.234\right)$ or hyporeactivity $\left(r=0.17, p_{\text {Bonferroni }}=0.750\right)$. There was also no meaningful or significant moderating effect of Group (all $p_{\text {Bonferroni }}>0.244$ ).

Thal GABA+ is associated with ADOS communication scores. We also explored whether there were any associations between autism severity and individual differences in metabolite concentration for each region. We identified an association between Thal GABA (when referenced to both water and creatine) and ADOS communication scores $\left(r=0.44, p_{\text {Bonferroni }}=0.081\right.$, $\mathrm{GABA}+/ \mathrm{Cr}: r=0.54, p_{\text {Bonferroni }}=0.022$ ), which we highlight in the Supplementary Fig. 5.

\section{Correlations between metabolite levels of SM1 and Thal with tactile sensitivity}

SM1 and Thal Glx with tactile perception. When collapsing across groups, there was evidence for linear associations between SM1 Glx levels and sequential frequency discrimination thresholds $\left(r=-0.22, p_{\text {Bonferroni }}=0.158\right.$; Fig. $\left.4 d\right)$ and the effect of simultaneity (i.e., the difference between simultaneous and sequential frequency discrimination thresholds; $r=0.27, p_{\text {Bonferroni }}=0.052$ ), though these associations did not survive correction for multiple 


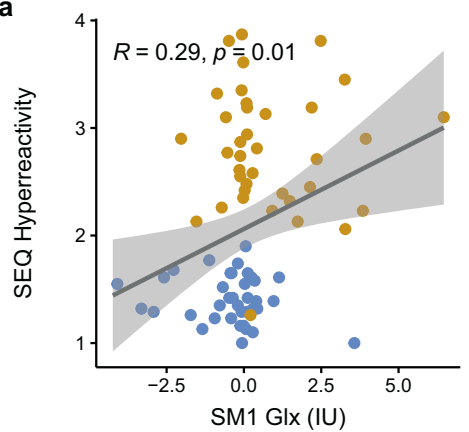

d

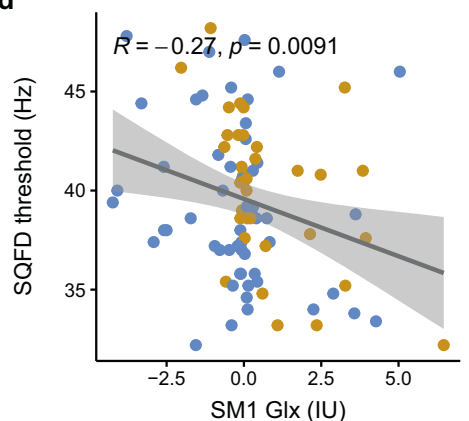

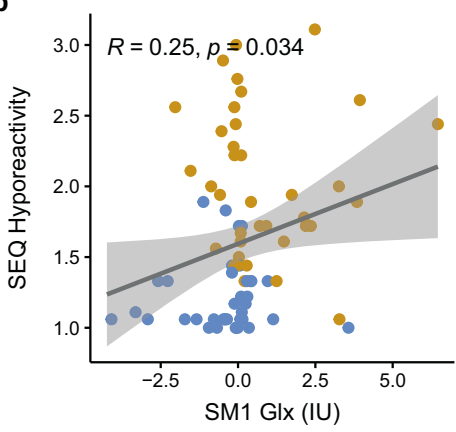

e

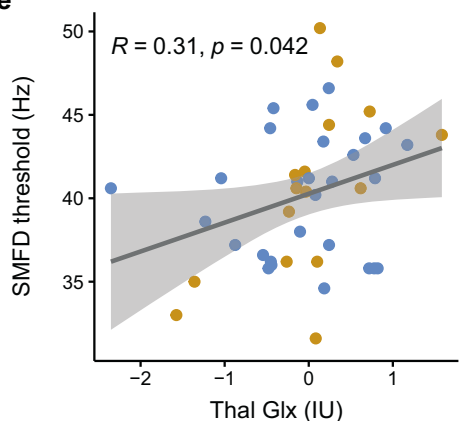

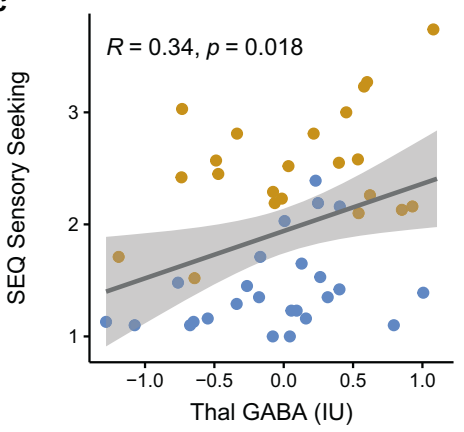

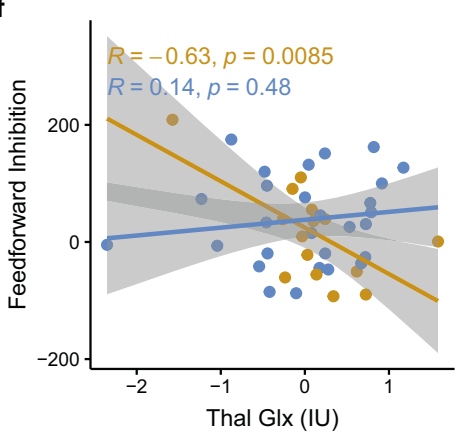

Fig. 4 Associations between GIx and GABA + of SM1 and Thal with sensory reactivity and tactile sensitivity. The ASD and TDC group are depicted as yellow and blue (respectively). Positive correlations were identified between Glx (IU) levels of SM1 and a Hyper- and b hyporeactivity scores on the SEQ. c Thal GABA (IU) levels also positively correlated with sensory seeking scores. d SM1 GIx (IU) levels showed evidence of a negative association to SQFD thresholds and e Thal Glx (IU) levels showed evidence of a positive association to SMFD thresholds. While we note that the associations presented in panel $\mathbf{d}$ and e do not survive correction for multiple comparisons, the associations were in the same direction when Glx was referenced to creatine, and the association in panel e was also stronger $(r=0.41, p=$ 0.0062), suggesting that these associations were neither simply due to the reference variable or spurious. See Supplementary Results and Supplementary Fig. 2.f There was a strong negative association between Thal Glx (IU) levels and feedforward inhibition in the ASD group that was otherwise absent in the control group. Indeed, a similar pattern of effect (i.e., an interaction effect showing a negative association between Thal Glx and feedforward inhibition in the ASD group that is otherwise absent in the TDC group) was also identified when Glx was referenced to creatine. See Supplementary Results - Fig. 2f. IU institutional units, Glx glutamate + glutamine, Cr creatine, SM1 primary sensorimotor cortex, Thal thalamus, ASD autism spectrum disorders, TDC typically developing controls. Note: although the correlations in panels a-d are driven by extreme values on either end, the variables are approximately normally distributed at the whole sample level. This suggests that these measures are accurately representing the continuum of possible scores on each of the measures. Note: $p$-values presented on all panels are not corrected for multiple comparisons, please refer to the $p$-values in the main text. The shaded area around the line of best fit represents the $95 \%$ confidence interval.

comparisons. There were no significant moderating effects of Group for any of the associations between SM1 Glx and tactile sensitivity (all $p_{\text {Bonferroni }}>0.885$ ).

For Thal Glx levels, group collapsed correlation analyses found an association between Thal Glx and simultaneous frequency discrimination thresholds $\left(r=0.31, p_{\text {Bonferroni }}=0.164\right.$; Fig. 4e), though this association also did not survive correction for multiple comparisons. Interestingly, however, there was some evidence for a moderating effect of Group on the association between Thal Glx and feedforward inhibition $\left[F(1,40)=7.35, p_{\text {Bonferroni }}=0.040, \mathrm{BF}_{10}\right.$ $=0.349$. Simple slope analyses revealed that while Thal Glx showed no association to feedforward inhibition in TDC $(r=0.14$, $\left.p_{\text {Bonferroni }}=0.960\right)$, there was a strong negative association between Thal Glx and feedforward inhibition in ASD $(r=-0.63$, $p_{\text {Bonferroni }}=0.018$; Fig. $4 f$ ).

No association between SM1 and Thal GABA+ with tactile perception. There were no significant associations between SM1 GABA + levels with any of the tactile perceptual thresholds (all $p_{\text {Bonferroni }}$ $>0.224$ ), nor was there evidence of a moderating effect of Group for any of the associations (all $p_{\text {Bonferroni }}>0.999$ ). Like SM1 GABA + , there were no significant associations between Thal GABA+ levels with any of the tactile perceptual thresholds (all $p_{\text {Bonferroni }}>$ 0.960), nor was there evidence of a moderating effect of Group (all $p_{\text {Bonferroni }}>0.999$ ).

\section{DISCUSSION}

In contrast to previous work showing altered GABA+ in ASD $[29,30,42,79,80]$, and in contrary to our hypotheses, GABA+ levels in both SM1 and Thal were comparable between children in the ASD and TDC groups. Instead, when compared to TDCs, Glx levels were significantly higher in the SM1 of the ASD group. Indeed, this finding could be interpreted as supporting the E-I balance theory of sensory symptoms in ASD (i.e., by increased 'excitation'). These results are also consistent with previous studies finding altered Glx levels in ASD [81-90]. We also identified a strong positive association between SM1 and Thal Glx in the ASD group that was otherwise absent in the TDC group, supporting the well-replicated and robust findings of hyperconnectivity between thalamic and sensory cortical regions in ASD [91-95].

While altered E-I balance has long been suggested to underlie the sensory symptoms of ASD, clear links between markers of excitation and inhibition to sensory symptoms of ASD were scarce. We took a comprehensive approach to linking excitation and inhibition to sensory symptoms, measuring both GABA+ and Glx levels as markers of E-I, as well as assessing sensory symptoms at both behavioral and perceptual levels. Behaviorally, our results revealed that children with higher Glx levels in SM1 were also those who were reported as being more hyper- and hyporeactive to sensory input. At the perceptual level, both SM1 and Thal GIx levels were loosely associated to differences in low-level tactile 
discrimination. Perhaps most strikingly, our results also revealed that children with ASD who had higher levels of Thal Glx also showed less feed-forward inhibition, a process known to be critical for preventing over-excitation and reducing task-irrelevant noise during signal processing [96].

When taken in the context of our previous work which had highlighted an association between low-level perceptual alterations and the symptoms of sensory hyper- and hyporeactivity in ASD [16], we can begin to construct a model of sensory abnormalities in ASD. First, elevated Glx levels, which is indirectly indicative of increased glutamatergic signaling, may explain greater difficulties with sensory discrimination and more difficulties with sensory gating. Due to these difficulties with discrimination and feedforward inhibition, individuals with ASD may experience the world as being noisier than their neurotypical counterparts. In turn, this may explain why individuals with ASD tend to be both hyper- and hyporeactive to sensory input. Indeed, noisier environments can be conducive to both greater levels of discomfort, as well as more difficulties with detecting relevant signals. Discomfort with the sensory environment can understandably result in hyperreactive responses to sensory input and difficulties with detecting relevant signals could feasibly explain hyporeactive responses since the lack of detection of relevant signals amongst noise could result in the absence of an otherwise expected response.

As always, we must consider these results in the context of the limitations of the study and its methods. First, we recognize that the ASD sample in the current study generally consist of individuals with 'high-functioning' autism. Moreover, the total sample consists of a narrow age range (by design) and is also predominantly male. These limitations do affect the generalizability of our findings. Next, while the E-I balance theory of ASD considers excitatory glutamatergic and inhibitory GABAergic signaling as independent processes, with regard to our MRS markers of both glutamate and GABA+, the picture is more complicated. As we have already discussed, our measures of glutamate are contaminated with glutamine. Amongst other roles [97-99], glutamine also functions as a precursor for the biosynthesis of GABA via glutamate [100], meaning that some of the Glx being measured represents glutamine and glutamate that function as precursors to GABA. Thus, while we consider measures of Glx and GABA+ as independent markers of excitation and inhibition respectively, MRS measures of Glx and GABA+ are interrelated. Moreover, and perhaps more critically, concentration levels do not directly reflect glutamatergic and GABAergic neurotransmission, but rather relate to total metabolite pools that certainly contribute to excitation and inhibition, but also have clear roles in energy and nitrogen metabolism [101]. Thus, while these pools contribute to $\mathrm{E}-\mathrm{I}$, they might reflect more general disruptions of Glu-GABA metabolism. Considering the importance of these limitations, we provide additional discussion of MRS-related methodological implication and how they might explain the discrepancies between the current and previous findings regarding GABA+ in the Supplementary Discussion.

In summary, the present study identified region-specific elevations of GIx in the SM1 of children with ASD. Individual differences in SM1 Glx levels were significantly associated with parent reports of hyperand hyporeactivity, as well as psychophysically derived measures of perceptual sensitivity in the tactile domain. Collectively, our findings provide strong evidence in support of the altered E-I balance theory of ASD. The results suggest that alterations at the neurobiological implementation of glutamatergic signaling may be related to alterations of tactile perception, which in turn may explain the observation of hyper- and hyporeactivity in ASD.

\section{REFERENCES}

1. Tomchek SD, Dunn W. Sensory processing in children with and without autism: a comparative study using the short sensory profile. Am J Occup Ther 2007;61:190-200.
2. Baranek GT, David FJ, Poe MD, Stone WL, Watson LR. Sensory Experiences Questionnaire: discriminating sensory features in young children with autism, developmental delays, and typical development. J Child Psychol Psychiatry Allied Discip. 2006;47:591-601.

3. Klintwall L, Holm A, Eriksson M, Carlsson LH, Olsson MB, Hedvall A, et al. Sensory abnormalities in autism. A brief report. Res Dev Disabil 2011;32:795-800.

4. Baker AEZ, Lane A, Angley MT, Young RL. The relationship between sensory processing patterns and behavioural responsiveness in autistic disorder: a pilot study. J Autism Dev Disord 2008;38:867-75.

5. Estes A, Zwaigenbaum L, Gu H, St John T, Paterson S, Elison JT, et al. Behavioral, cognitive, and adaptive development in infants with autism spectrum disorder in the first 2 years of life. J Neurodev Disord 2015;7:24.

6. Robertson CE, Baron-Cohen S. Sensory perception in autism. Nat Rev Neurosci. 2017;18:671-84.

7. Yeung $\mathrm{LHJ}$, Thomacos N. Assessments of sensory processing in infants and children with autism spectrum disorder between 0-12 years old: a scoping review. Res Autism Spectr Disord. 2020;72:101517.

8. Suarez MA. Sensory processing in children with autism spectrum disorders and impact on functioning. Pediatr Clin North Am. 2012;59:203-14.

9. Rogers SJ, Ozonoff S. Annotation: what do we know about sensory dysfunction in autism? A critical review of the empirical evidence. J. Child Psychol Psychiatry. 2005;46:1255-68.

10. Green SA, Hernandez L, Tottenham N, Krasileva K, Bookheimer SY, Dapretto M. Neurobiology of sensory overresponsivity in youth with autism spectrum disorders. JAMA Psychiatry. 2015;72:778-86.

11. Cheung PPP, Lau BWM. Neurobiology of sensory processing in autism spectrum disorder. Prog Mol Biol Transl Sci. 2020;173:161-81.

12. Brown T. Sensory Processing Measure. Encyclopedia of Autism Spectrum Disorders. Springer; 2018. p. 1-10.

13. Tavassoli T, Hoekstra RA, Baron-Cohen S. The Sensory Perception Quotient (SPQ): development and validation of a new sensory questionnaire for adults with and without autism. Mol Autism. 2014;5:29.

14. Siper PM, Kolevzon A, Wang AT, Buxbaum JD, Tavassoli T. A clinician-administered observation and corresponding caregiver interview capturing DSM-5 sensory reactivity symptoms in children with ASD. Autism Res. 2017;10:1133-40.

15. Ben-Sasson A, Gal E, Fluss R, Katz-Zetler N, Cermak SA. Update of a meta-analysis of sensory symptoms in ASD: a new decade of research. J Autism Dev Disord2019;49:4974-96

16. He JL, Wodka E, Tommerdahl M, Edden R, Mikkelsen M, Mostofsky SH, et al. Disorder-specific alterations of tactile sensitivity in neurodevelopmental disorders. Commun Biol. 2021;4:97.

17. Stevens SS. The Psychophysics of Sensory Function. Sens Commun. 2013. https://doi.org/10.7551/mitpress/9780262518420.003.0001.

18. Read JCA. The place of human psychophysics in modern neuroscience. Neuroscience. 2015;296:116-29.

19. Puts NAJ, Wodka EL, Tommerdahl M, Mostofsky SH, Edden RAE. Impaired tactile processing in children with autism spectrum disorder. J Neurophysiol 2014;111:1803-11.

20. Bryant LK, Woynaroski TG, Wallace MT, Cascio CJ. Self-reported sensory hypersensitivity moderates association between tactile psychophysical performance and autism-related traits in neurotypical adults. J Autism Dev Disord 2019;49:3159-72.

21. Quinde-Zlibut JM, Okitondo CD, Williams ZJ, Weitlauf A, Mash LE, Heflin BH, et al. Elevated thresholds for light touch in children with autism reflect more conservative perceptual decision-making rather than a sensory deficit. Front Hum Neurosci 2020;14:122.

22. Cascio C, McGlone F, Folger S, Tannan V, Baranek G, Pelphrey KA, et al. Tactile perception in adults with autism: A multidimensional psychophysical study. J Autism Dev Disord 2008;38:127-37.

23. Schallmo M-P, Kale A, Kolodny T, Millin R, Bernier R, Murray S. Weaker neural suppression in autism spectrum disorder. J Vis. 2018;18:548.

24. Hadad B, Schwartz S, Nizri O, Harel N. Atypical basic psychophysics in autism: violation of Weber's law in vision and haptic. J Vis. 2018;18:35

25. O'Riordan M, Passetti F. Discrimination in autism within different sensory modalities. J Autism Dev Disord 2006;36:665-75.

26. Bonnel A, Mottron L, Peretz I, Trudel M, Gallun E, Bonnel AM. Enhanced pitch sensitivity in individuals with autism: a signal detection analysis. J Cogn Neurosci 2003;15:226-35.

27. Jones CR, Happé F, Baird G, Simonoff E, Marsden AJ, Tregay J, et al. Auditory discrimination and auditory sensory behaviours in autism spectrum disorders. Neuropsychologia. 2009;47:2850-8.

28. Stanutz S, Wapnick J, Burack JA. Pitch discrimination and melodic memory in children with autism spectrum disorders. Autism. 2014;18:137-47.

29. Pizzarelli R, Cherubini E. Alterations of GABAergic signaling in autism spectrum disorders. Neural Plasticity. 2011;2011:297153. 
30. Puts N, Wodka EL, Harris AD, Crocetti D, Tommerdahl M, Mostofsky SH, et al. Reduced GABA and altered somatosensory function in children with autism spectrum disorder. Autism Res. 2017;10:608-19.

31. Horder J, Petrinovic MM, Mendez MA, Bruns A, Takumi T, Spooren W, et al. Glutamate and GABA in autism spectrum disorder-a translational magnetic resonance spectroscopy study in man and rodent models. Transl Psychiatry. 2018;8:106.

32. Fatemi SH, Folsom TD, Reutiman TJ, Thuras PD. Expression of GABAB receptors is altered in brains of subjects with autism. Cerebellum. 2009;8:64-9.

33. O'Connor K. Auditory processing in autism spectrum disorder: a review. Neurosci Biobehav Rev. 2012;36:836-54.

34. Simmons DR, Robertson AE, McKay LS, Toal E, McAleer P, Pollick FE. Vision in autism spectrum disorders. Vis Res. 2009;49:2705-39.

35. Mallet N, Le Moine C, Charpier S, Gonon F. Feedforward inhibition of projection neurons by fast-spiking GABA interneurons in the rat striatum in vivo. J Neurosci 2005;25:3857-69.

36. Herrera CG, Cadavieco MC, Jego S, Ponomarenko A, Korotkova T, Adamantidis A. Hypothalamic feedforward inhibition of thalamocortical network controls arousal and consciousness. Nat Neurosci 2016;19:290-8.

37. Sloviter RS. Feedforward and feedback inhibition of hippocampal principal cell activity evoked by perforant path stimulation: GABA-mediated mechanisms that regulate excitability In Vivo. Hippocampus. 1991;1:31-40.

38. Jacob TC, Moss SJ, Jurd R. GABAA receptor trafficking and its role in the dynamic modulation of neuronal inhibition. Nat Rev Neurosci. 2008;9:331-43.

39. Coghlan S, Horder J, Inkster B, Mendez MA, Murphy DG, Nutt DJ. GABA system dysfunction in autism and related disorders: from synapse to symptoms. Neurosci Biobehav Rev. 2012;36:2044-55.

40. Hussman JP. Suppressed gabaergic inhibition as a common factor in suspected etiologies of autism [1]. J Autism Dev Disord. 2001;31:247-8.

41. Rubenstein JLR, Merzenich MM. Model of autism: Increased ratio of excitation/ inhibition in key neural systems. Genes Brain Behav. 2003;2:255-67.

42. Sohal VS, Rubenstein JLR. Excitation-inhibition balance as a framework for investigating mechanisms in neuropsychiatric disorders. Mol Psychiatry. 2019;24:1248-57.

43. Lee $\mathrm{E}$, Lee J, Kim E. Excitation/Inhibition imbalance in animal models of autism spectrum disorders. Biol Psychiatry. 2017;81:838-47.

44. de Graaf RA. In Vivo NMR Spectroscopy: Principles and Techniques. 2nd ed. John Wiley and Sons, Ltd; 2007.

45. Mullins PG, McGonigle DJ, O'Gorman RL, Puts NA, Vidyasagar R, Evans CJ, et al. Current practice in the use of MEGA-PRESS spectroscopy for the detection of GABA. Neurolmage. 2014;86:43-52.

46. Mullins PG, Chen H, Xu J, Caprihan A, Gasparovic C. Comparative reliability of proton spectroscopy techniques designed to improve detection of J-coupled metabolites. Magn Reson Med 2008;60:964-9.

47. Schür RR, Draisma LW, Wijnen JP, Boks MP, Koevoets MG, Joëls $M$, et al. Brain GABA levels across psychiatric disorders: A systematic literature review and meta-analysis of 1H-MRS studies. Hum Brain Mapp. 2016;37:3337-52.

48. Ajram LA, Pereira AC, Durieux A, Velthius HE, Petrinovic MM, McAlonan GM. The contribution of $[1 \mathrm{H}]$ magnetic resonance spectroscopy to the study of excitation-inhibition in autism. Prog Neuro-Psychopharmacol Biol Psychiatry. 2019:89:236-44.

49. Ferezou I, Haiss F, Gentet LJ, Aronoff R, Weber B, Petersen CC. Spatiotemporal dynamics of cortical sensorimotor integration in behaving mice. Neuron. 2007;56:907-23.

50. Petersen $\mathrm{CCH}$. Sensorimotor processing in the rodent barrel cortex. Nat Rev Neurosci. 2019;20:533-46.

51. Nicolelis MAL, Baccala LA, Lin RCS, Chapin JK. Sensorimotor encoding by synchronous neural ensemble activity at multiple levels of the somatosensory system. Science. 1995;268:1353-8.

52. Davis KD, Kwan CL, Crawley AP, Mikulis DJ. Functional MRI study of thalamic and cortical activations evoked by cutaneous heat, cold and tactile stimuli. J Neurophysiol 1998;80:1533-46.

53. Mountcastle VB, Henneman E. The representation of tactile sensibility in the thalamus of the monkey. J Comp Neurol 1952;97:409-39.

54. Ghazanfar AA, Stambaugh CR, Nicolelis MAL. Encoding of tactile stimulus location by somatosensory thalamocortical ensembles. J Neurosci. 2000;20:3761-75.

55. American Psychiatric Association. DSM V. J Dev Behav Pediatr. 2013. https://doi. org/10.1097/DBP.0b013e3182978a90.

56. Kim SH, Lord C. Encyclopedia of Clinical Neuropsychology. Springer; 2018. p. 432-6.

57. Lord C, Rutter M, Le Couteur A. Autism Diagnostic Interview-Revised: a revised version of a diagnostic interview for caregivers of individuals with possible pervasive developmental disorders. J Autism Dev Disord 1994;24:659-85.

58. Dumont R, Willis JO, Veizel K, Zibulsky J. Wechsler Adult Intelligence Scale. 4th ed. Encyclopedia of Special Education. 2014.
59. Weiss LG, Locke V, Pan T, Harris JG, Saklofske DH, Prifitera A. WISC-V. 5th ed. SAGE publishing; 2019. p. 129-95.

60. Oldfield RC. The assessment and analysis of handedness: the Edinburgh inventory. Neuropsychologia. 1971;9:97-113.

61. Yousry TA, Schmid UD, Alkadhi H, Schmidt D, Peraud A, Buettner A, et al. Localization of the motor hand area to a knob on the precentral gyrus. A new landmark. Brain. 1997;120:141-57.

62. Tkáč I, Starčuk Z, Choi IY, Gruetter R. In vivo 1H NMR spectroscopy of rat brain at 1 ms echo time. Magn Reson Med. 1999;41:649-56.

63. Edden RAE, Puts NAJ, Harris AD, Barker PB, Evans CJ. Gannet: a batch-processing tool for the quantitative analysis of gamma-aminobutyric acid-edited MR spectroscopy spectra. J Magn Reson Imaging. 2014. https://doi.org/10.1002/jmri.24478.

64. Penny W, Friston K, Ashburner J, Kiebel S, Nichols T. Statistical parametric mapping: the analysis of functional brain images. 2007. https://doi.org/10.1016/ B978-0-12-372560-8.X5000-1.

65. Harris AD, Puts NAJ, Edden RAE. Tissue correction for GABA-edited MRS: considerations of voxel composition, tissue segmentation, and tissue relaxations. J Magn Reson Imaging. 2015. https://doi.org/10.1002/jmri.24903.

66. Porges EC, Woods AJ, Lamb DG, Williamson JB, Cohen RA, Edden R, et al. Impact of tissue correction strategy on GABA-edited MRS findings. Neuroimage. 2017;162:249-56.

67. Dunn W. The sensations of everyday life: Empirical, theoretical, and pragmatic considerations. Am J Occup Ther. 2001. https://doi.org/10.5014/ajot.55.6.608.

68. Puts NAJ, Edden RAE, Wodka EL, Mostofsky SH, Tommerdahl M. A vibrotactile behavioral battery for investigating somatosensory processing in children and adults. J Neurosci Methods. 2013. https://doi.org/10.1016/j.jneumeth.2013.04.012.

69. $\mathrm{R}$ Core Team. R: A language and environment for statistical computing. $\mathrm{R}$ Foundation for Statistical Computing. Vienna, Austria: 2018. https://doi.org/ 10.1108/eb003648.

70. Ben-Shachar M, Lüdecke D, Makowski D. Effectsize: estimation of effect size indices and standardized parameters. J Open Source Softw. 2020. https://doi. org/10.21105/joss.02815.

71. Morey RD, Rouder JN, Jamil T. Package ' BayesFactor'. R Packag. version 0.9.12-2 (2015).

72. Ly A, Verhagen J, Wagenmakers EJ. Harold Jeffreys's default Bayes factor hypothesis tests: explanation, extension, and application in psychology. J Math Psychol 2016;72:19-32.

73. Leys C, Ley C, Klein O, Bernard P, Licata L. Detecting outliers: do not use standard deviation around the mean, use absolute deviation around the median. J Exp Soc Psychol 2013. https://doi.org/10.1016/j.jesp.2013.03.013.

74. Green SA, Hernandez L, Bookheimer SY, Dapretto M. Reduced modulation of thalamocortical connectivity during exposure to sensory stimuli in ASD. Autism Res. 2017. https://doi.org/10.1002/aur.1726.

75. Nair A, Treiber JM, Shukla DK, Shih P, Müller RA. Impaired thalamocortical connectivity in autism spectrum disorder: a study of functional and anatomical connectivity. Brain. 2013. https://doi.org/10.1093/brain/awt079.

76. Khan S, Hashmi JA, Mamashli F, Bharadwaj HM, Ganesan S, Michmizos KP, et al. Altered onset response dynamics in somatosensory processing in autism spectrum disorder. Front Neurosci 2016;10:255.

77. Kharazia VN, Weinberg RJ. Glutamate in thalamic fibers terminating in layer IV of primary sensory cortex. J Neurosci 1994;14:6021-32.

78. Makin TR, De Xivry JJO. Ten common statistical mistakes to watch out for when writing or reviewing a manuscript. Elife. 2019;8:e48175.

79. Harada M, Taki MM, Nose A, Kubo H, Mori K, Nishitani $H$, et al. Non-invasive evaluation of the GABAergic/glutamatergic system in autistic patients observed by MEGA-editing proton MR spectroscopy using a clinical 3 Tesla instrument. J. Autism Dev. Disord. 2011:41:447-54.

80. Hashemi E, Ariza J, Rogers H, Noctor SC, Martínez-Cerdeño V. The number of parvalbumin-expressing interneurons is decreased in the medial prefrontal cortex in autism. Cereb Cortex. 2017. https://doi.org/10.1093/cercor/bhw021.

81. Zheng Z, Zhu T, Qu Y, Mu D. Blood glutamate levels in autism spectrum disorder: a systematic review and meta-analysis. PLoS ONE. 2016. https://doi.org/ 10.1371/journal.pone.0158688.

82. Naaijen J, Lythgoe DJ, Amiri H, Buitelaar JK \& Glennon JC. Fronto-striatal glutamatergic compounds in compulsive and impulsive syndromes: a review of magnetic resonance spectroscopy studies. Neurosci Biobehav Rev. 2015. https:// doi.org/10.1016/j.neubiorev.2015.02.009.

83. Khalifa D, Shahin O, Salem D, Raafat O. Serum glutamate was elevated in children aged 3-10 years with autism spectrum disorders when they were compared with controls. Acta Paediatr Int J Paediatr. 2019. https://doi.org/10.1111/ apa.14477.

84. Naaijen J, Zwiers MP, Glennon JC, Franke B, Buitelaar JK, Lythgoe DJ. Elevated glutamate levels in anterior cingulate cortex in paediatric compulsive syndromes: a multi-centre magnetic resonance spectroscopy study. Eur Neuropsychopharmacol 2016;26:S720. 
85. Brown MS, Singel D, Hepburn S, Rojas DC. Increased glutamate concentration in the auditory cortex of persons with autism and first-degree relatives: A 1H-MRS study. Autism Res. 2013;6:1-10.

86. Horder J, Lavender T, Mendez MA, O'Gorman R, Daly E, Craig MC, et al. Reduced subcortical glutamate/glutamine in adults with autism spectrum disorders: A [1H]MRS study. Transl Psychiatry. 2013;3:279.

87. Joshi G, Biederman J, Wozniak J, Goldin RL, Crowley D, Furtak S, et al. Magnetic resonance spectroscopy study of the glutamatergic system in adolescent males with high-functioning autistic disorder: a pilot study at 4T. Eur Arch Psychiatry Clin Neurosci 2013;263:379-84.

88. Kubas B, Kułak W, Sobaniec W, Tarasow E, Lebkowska U, Walecki J. Metabolite alterations in autistic children: a $1 \mathrm{H}$ MR spectroscopy study. Adv Med Sci 2012;57:152-6.

89. Wheeler DC, Morgan R, Thomas DM, Seed M, Rees A, Moore RH. In vivo $1 \mathrm{H}-$ magnetic resonance spectroscopy study of amygdala-hippocampal and parietal regions in autism. Am J Psychiatry. 2006;9:221-6.

90. Tebartz Van Elst L, Maier S, Fangmeier T, Endres D, Mueller GT, Nickel K, et al. Disturbed cingulate glutamate metabolism in adults with high-functioning autism spectrum disorder: evidence in support of the excitatory/inhibitory imbalance hypothesis. Mol. Psychiatry. 2014;19:1314-25.

91. Supekar K, Uddin LQ, Khouzam A, Phillips J, Gaillard WD, Kenworthy LE, et al. Brain hyperconnectivity in children with autism and its links to social deficits. Cell Rep. 2013;5:738-47.

92. Martínez K, Martínez-García M, Marcos-Vidal L, Janssen J, Castellanos FX, Pretus $C$, et al. Sensory-to-cognitive systems integration is associated with clinical severity in autism spectrum disorder. J Am Acad Child Adolesc Psychiatry. 2020;59:422-33.

93. Fu Z, Tu Y, Di X, Du Y, Sui J, Biswal BB, et al. Transient increased thalamic-sensory connectivity and decreased whole-brain dynamism in autism. Neurolmage. 2019;190:191-204.

94. Smith GL, Goulbourn RA, Burt RA, Chatfield DH. Reproducible functional connectivity alterations are associated with autism spectrum disorder. bioRxiv. 2018;4:585-90.

95. Di Martino A, O'Connor D, Chen B, Alaerts K, Anderson JS, Assaf M, et al. Enhancing studies of the connectome in autism using the autism brain imaging data exchange II. Sci Data. 2017;4:1-15.

96. Khubieh A, Ratté S, Lankarany M, Prescott SA. Regulation of cortical dynamic range by background synaptic noise and feedforward inhibition. Cereb. Cortex. 2016. https://doi.org/10.1093/cercor/bhv157.

97. Zhou Y, Danbolt NC. Glutamate as a neurotransmitter in the healthy brain. J Neural Transm. 2014. https://doi.org/10.1007/s00702-014-1180-8.

98. Javitt DC, Schoepp D, Kalivas PW, Volkow ND, Zarate C, Merchant K, et al. Translating glutamate: from pathophysiology to treatment. Sci Transl Med. 2011;3:102.

99. Willard SS, Koochekpour S. Glutamate, glutamate receptors, and downstream signaling pathways. Int J Biol Sci. 2013. https://doi.org/10.7150/ijbs.6426.

100. Bak LK, Schousboe A, Waagepetersen HS. The glutamate/GABA-glutamine cycle: aspects of transport, neurotransmitter homeostasis and ammonia transfer. J Neurochem. 2006. https://doi.org/10.1111/j.1471-4159.2006.03913.x.
101. Rae C, Nasrallah FA, Balcar VJ, Rowlands BD, Johnston GA, Hanrahan JR. Metabolomic approaches to defining the role(s) of GABAp receptors in the brain. J Neuroimmune Pharmacol 2015;10:445-56.

\section{ACKNOWLEDGEMENTS}

This work was funded by NIH/NIMH R21MH098228, R01MH106564, R01MH078160, R00MH107719 and R21HD100869 and used resources provided by P41EB031771. J.L. H., E.L.W., and N.A.J.P. received salary support from the Nancy Lurie Marks Family Foundation as part Autism Sensory Research Consortium. S.H.M. receives royalties for US Patent 10,410,041. We would like to thank all the participants and their families who participated in the studies from which these data were acquired. We would also like to thank all the research coordinators and assistants that helped collect these data at various stages.

\section{COMPETING INTERESTS}

The authors declare no competing interests.

\section{ADDITIONAL INFORMATION}

Supplementary information The online version contains supplementary material available at https://doi.org/10.1038/s41398-021-01525-1.

Correspondence and requests for materials should be addressed to N.A.J.P.

Reprints and permission information is available at http://www.nature.com/ reprints

Publisher's note Springer Nature remains neutral with regard to jurisdictional claims in published maps and institutional affiliations.

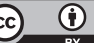

Open Access This article is licensed under a Creative Commons Attribution 4.0 International License, which permits use, sharing, adaptation, distribution and reproduction in any medium or format, as long as you give appropriate credit to the original author(s) and the source, provide a link to the Creative Commons license, and indicate if changes were made. The images or other third party material in this article are included in the article's Creative Commons license, unless indicated otherwise in a credit line to the material. If material is not included in the article's Creative Commons license and your intended use is not permitted by statutory regulation or exceeds the permitted use, you will need to obtain permission directly from the copyright holder. To view a copy of this license, visit http://creativecommons. org/licenses/by/4.0/

(c) The Author(s) 2021 